\title{
PAST, PRESENT, AND FUTURE OF PHOTOGRAPHIC SURVEYS
}

\author{
Alain Maury \\ Télescope de Schmidt O.A.M.II.N.S.U. \\ St Vallier de Thiey - 06460 \\ France
}

\begin{abstract}
Surveying the entire sky requires very efficient instrumentation, if the project has to be done in a relatively short period. In this is a review of the existing photographic surveys, it is demonstrated that photography, because of its very large format, is still the best existing technique for survey purposes.
\end{abstract}

\section{1 - How to compare detectors for optlcal surveys?}

The factor to be taken into account is the time it takes to survey the sky to a given limiting magnitude, in a given spectral band and with a given angular resolution. This factor is itself a compromise between many other factors. Spectral bands are usually determined by colored filters with a width of about $100 \mathrm{~nm}$ to $150 \mathrm{~nm}$. The parameters to be taken into account are: the telescope's field of view (detector size ); the sensitivity of the detector, expressed as the necessary exposure time to obtain a given photometric precision (DQE and focal ratio and diameter of the telescope); the image sampling quality or FTM of the system, which is a function of the detector's pixel size, the focal length of the telescope, its optical quality, and the mean seeing conditions at the site. Also to be taken into account is the dynamic range of the detector ( ratio of the saturation value of the detector and the sky value needed to produce a given $S / N$ ). A general "factor of efficiency" defined by R. Angel in 1983 is:

$$
(A \Omega) \text { eff }=\frac{\pi * a * D Q E}{4 * F^{2}}
$$

where $a$ is the size of the detector in square centimeters, and $F$ is the focal ratio of the telescope. This expression does not take angular resolution into

account, but it does provide a first approximation to efficiencies of survey instruments. We will use it here.

\section{Evolution of photographic surveys.}

\section{6 vears ago..}

In 1881, the Henry brothers made a $16 \mathrm{~cm}$ F/13 astrophotographic doublet which gave images good enough to convince Admiral Mouchez of the technical feasibility of a photographic "Carte du Ciel". This first prototype lens was lost, but in 1982, I was

lucky enough to buy a doublet of about the same type as this lens. It is a $147 \mathrm{~mm}$ aperture doublet, with a focal length of about $2700 \mathrm{~mm}$, yielding a relative aperture of F/19. An engraving on the front lens reads: "Objectif Photographique Henry Fres Paris 
1881 ". In 1984, Mr Texereau, then at CERGA made an optical analysis of this doublet and told me that it might, in fact, be the prototype lens. I bought this doublet from a priest who told me that he bought it at the flea market in Paris in 1950 (!), so it is not possibile to trace its history... nor is there any chance of ever finding original plates taken with it. Whether or not this is the original prototype lens remains an open question.

The Carte du Ciel is an important milestone in the history of astronomical photography and astronomy: Use of precision equatorial mounts, photographic plates for astrometric purposes, specially corrected optics... and survey of the whole sky.

\section{7 years ago...}

Bernhardt Schmidt invented his telescope. This invention eventually led to the Palomar 18 inch and to the 48 inch Schmidt telescope, which was used for the first Palomar Sky Survey. The basic ingredients of this Survey were: a large aperture optical system; two colors, large, reciprocity failure corrected photographic plates; tray rocking processing and the survey of $72 \%$ of the whole sky completed in a decade.

\section{Today.}

The Second Palomar Sky Survey is under way using the 48 inch Schmidt. It is not substantially different of the SRC/ESO Southern Sky Surveys: The use of three passbands; hypersensitised fine grain plates; achromatic doublet corrector. It is not important here to talk further about the possibilities offered by such an instrumentation: See for example the paper by Dr. Reid in this volume.

\section{Tomorrow.e}

Bigger classical Schmidt telescopes will probably never be built. Future wide field telescopes will very probably use reflective optics on altazimuth mounts, used both for wide field imaging and fiber optics spectroscopy. Such a design is the Mersenne Schmidt telescope. Also, many astronomers feel that photographic plate has reached the limit of their usefulness, and that, because of its low DQE, it will be replaced by CCD detectors.

Table 1: Evolution of photographlc survey with medlum slze telescopes:

For the Carte du Ciel lens, the DQE is supposed to have been in the $0.01 \%$ range. For PSS 1 , it was around $0.1 \%$, and around $1 \%$ for PSS 2. All other figures are for a DQE of $5 \%$ ( which is what future plates will certainly reach when exposed to the peak $S / N$ density).

\begin{tabular}{|c|c|c|c|c|c|c|}
\hline Optic & size & Field & Plates & Scale & Efficiency & Instrument Type \\
\hline $.33 m$ & $f / 10$ & $2.7^{\circ}$ & $16 \mathrm{~cm}$ & $60.1^{11} / \mathrm{mm}$ & $0.0002 \mathrm{~cm} 2$ & $\mathrm{Ciel}$ \\
\hline $1.2 r$ & $f / 2.5$ & $6.5^{\circ}$ & $35 \mathrm{~cm}$ & $67.3^{\mathrm{n}} / \mathrm{mm}$ & $0.15 \mathrm{~cm} 2$ & $1103 a O$ \\
\hline $1.2 \mathrm{~m}$ & $f / 2.5$ & $6.5^{\circ}$ & $35 \mathrm{~cm}$ & $67.3^{\mathrm{n} / \mathrm{mm}}$ & $1.5 \mathrm{~cm} 2$ & PSS 2 IllaJ \\
\hline $1.2 \mathrm{~m}$ & $f / 2.5$ & $6.5^{\circ}$ & $35 \mathrm{~cm}$ & $67.3^{\mathrm{n}} / \mathrm{mm}$ & $7.7 \mathrm{~cm} 2$ & New emulsions \\
\hline
\end{tabular}

\section{New trends In astronomical photography.}

A new system of plate processing machine called a "grid processing machine" was recently invented by Dumoulin and Quebatte at the Sky Atlas Laboratory of ESO. It is able to process photographic plates to a level of uniformity and efficiency not previously obtainable ( 3 times more uniform than an optimally tuned tray rocker, and 
with an increase of apparent speed and contrast ). This means that a lllaJ could be exposed only 40 minutes at the telescope instead of the current 50 to 60 minutes, while having a better uniformity and contrast than with a tray rocker.

A second point is the future availability of improved emulsions. Emulsions with a DQE in the range of 8 to $10 \%$ can be produced and will, hopefully in the near future, be adapted by Kodak to the specific requirements of astronomical photography: Low light level, and hydrogen hypersensitisation. These plates will have the same effective speed as IllaJ emulsions. Using $T$ grain technology, it is possible to obtain an emulsion which has a much more linear characteristic curve than a IllaJ. The maximum signal to noise ratio then occurs at a lower density than with the IIlaJ, the DQE curve is almost coincident with the S/N curve. This offers the advantage of optimising the efficiency of the emulsion for actual exposure conditions: A lllaJ emulsion, for example, may have a peak DQE of $4 \%$ but, at the peak $S / N$ density, the DQE is below the $1 \%$ point. This solution also allows for a very broad $S / N$ curve. Another solution is to improve the existing IIlaJ and create a monodisperse emulsion which has all the basic characteristics of the IIlaJ ( speed, contrast and shape of the characteristic curve ) except a finer grain. The peak $S / N$ can be expected to be 80 to 100 . This means reaching one magnitude deeper than is now possible.

\section{CCD chips as survey detectors}

CCD cameras have a very small field ( typically 3 orders of magnitude smaller than a Schmidt plate ), poor resolution ( a lllaJ emulsion resolves 200 lines per $\mathrm{mm}$, or 5 to 10 times more than a typical CCD chip), only medium dynamic range in sky limited operation (when the sky level is brought to a $\mathrm{S} / \mathrm{N}$ of 50 ( 2500 electrons for the sky level), the dynamic range is less than 100 ), and archival quality is limited to the lifetime of computer technology, which is usually 5 times less than a correctly processed photographic plate. The true DQE, even if many times better than the photographic emulsion, is much lower than most astronomers think. The limitation is the silicon reflectivity characteristics (below $64 \%$ at the best when most astronomers think CCD's can have a DQE of $80 \%$ !), the charge transfer problems, and the need for band filters, which do not have good transmission characteristics ( $60 \%$ peak ). At the best, the total efficiency (electrons recorded divided by the available photon flux at the focal plane of the telescope) of a CCD camera is below $30 \%$ in the red, and below $5 \%$ in the blue, while the field is typically 2 orders of magnitude smaller than photographic plates.

The future of large CCD chips ( able to compete with the photographic plate for survey purposes ) is very uncertain as no astronomical grade chips larger than 1000*1000 have been "mass-produced" since 1982. It is very probable that they will not reach the 100 megapixels capacity by the end of the century, if they ever do.

\section{Deep. limited surveys with near future telescopes}

The new generation of 8 to 16 meter telescopes now being designed, do not incorporate photography as a likely detector. The design for the ESO VLT is a good example: its focal ratios of $F / 15$ is completely impractical for photography; 30 arc minutes field ( $1.05 \mathrm{~m}$ diameter ) which will certainly be usable for fiber optic spectrography, but not for wide field imaging. The scale at this focus is 1.72 arc second per millimeter, which is even too large for CCD imaging when the seeing disk is larger than $0.25^{\prime \prime}$. Bringing the focal ratio to one half of this value would give $F / 7.5$, and a field of $50 \mathrm{~cm}$ ( for which both photographic plates and measuring machines do already exist!). Using the prime focus with a triplet corrector giving a $1^{\circ}$ field of view with an image quality better than 1 arc second is an even better solution, but seems to be a rather low 
priority. Covering 1 or 4 full square degree in two hours ( using the four telescopes on four adjacent fields) down to magnitude 26 does not seem to interest European astronomers.

Iable 2: ESO VLI telescopes used with photography

\begin{tabular}{|c|c|c|c|c|c|}
\hline Optics size & Field & Plates & Scale & Efficiency & Instrument Type \\
\hline$n * 4 \quad f / 2$ & $2^{\circ}$ & $44^{\star 2} 28 \mathrm{~cm}$ & $12.9^{\mathrm{m}} / \mathrm{mm}$ & $30.8 \mathrm{~cm} 2$ & PF \\
\hline $8 m * 4 \quad f / 7.5$ & $1^{\circ}$ & $4 * 50 \mathrm{~cm}$ & $3.44 \mathrm{n} / \mathrm{mm}$ & $7 \mathrm{~cm} 2$ & ESO VLT bis \\
\hline
\end{tabular}

\section{Future surveys.}

As more powerful telescopes are put into operation ( for example the Keck 10 meter, the US NNTT, the ESO VLT, the Hubble Space Telescope), astronomers will be able to reach fainter and fainter limits ( mag. 28/29 is expected before the end of the century ), and the difference in limiting magnitude between the existing surveys and the observational possibilities will become too large. Optical surveys are still fundamental to astronomy, and even if it is possible to take a spectrum of a 23rd magnitude object, this object must first be discovered. Survey followed by precise analysis of only the interesting objects discovered is still a very powerful method of astronomical discovery. Because the photographic plate has to survey and discover objects which will be examined closely with detectors with better photometric quality, the survey instrument of the future will need to be as large as the spectrographic instrument. Recent optical design allow to create such a versatile instrument. The Mersenne Schmidt telescope could survey the sky down to magnitude 25 , and then take spectra using a 1000 fiber optics CCD spectrograph ( or 4*250 fiber optics spectrographs ). An instrument of the same basic design, but operated from space would present ideal characteristics: Discovery of new frequency domains ( 3 new passbands in the UV ), higher resolution ( limited to the optics and the guidance system ), darker skies under continuous operation conditions ( no days, no clouds, no bad seeing ), and weightless environment ( which would greatly ease the use of large, very fast optical systems).

Table 3: Future ground based surveys, "analog" versus "dlgital" detectors. Analog: $60 \mathrm{~cm}$ wide photographic plate, with a DQE of $5 \%$

Digital: Matrix of $6 \times 6$ chips (each separated and shifted by a 6 th of a chip ) $=36$ chips.- $20 \%$ DQE, $6.1^{\star} 6.1 \mathrm{~cm}$ chips, $74 \%$ filling factor

- Best seeing of $0.5^{\prime \prime}$, each $30 \mu \mathrm{m}$ pixel $=0.125^{\prime \prime} \Rightarrow$ F.L. $=49.5 \mathrm{~m} \Rightarrow 1^{\circ}=864 \mathrm{~mm}$

$\begin{array}{llllll}\text { Optics size } & \text { Field } & \text { Plates } & \text { Scale } & \text { Efficiency } & \text { Instrument Type } \\ 5.2 \mathrm{~m} \mathrm{f} / 1.6 & 4.1^{\circ} & 60 \mathrm{~cm} & 24.8^{\mathrm{n}} / \mathrm{mm} & 55 \mathrm{~cm} 2 & \text { Mersenne-Schmidt } \\ 5 \mathrm{~m} \mathrm{f} / 10 & .5^{\circ} & 43.2 \mathrm{~cm} & 4.2^{\mathrm{N}} / \mathrm{mm} & 2.1 \mathrm{~cm} 2 & \text { CCD imager } \\ 8 \mathrm{~m} \mathrm{f} / 6.2 & .5^{\circ} & 43.2 \mathrm{~cm} & 4.2^{\mathrm{n}} / \mathrm{mm} & 5.5 \mathrm{~cm} 2 & \end{array}$

\section{Conclusion:}

The surveys of the future will require a higher efficiency than currently available with the existing instruments and detectors. It seems that photographic plates will be improved in the near future, and combining these new emulsions with adequate 
processing techniques will allow a higher DQE to be reached. In the blue region of the spectrum, the photographic plate might even be better than some CCD's ( in sky limited, broad band imagery ). Such an instrument would easily outperform any CCD based system using any foreseable technology.

Ground based telescopes provide a first solution: However, the atmosphere still limits the resolution. The operating costs will be relatively low, even if two instruments have to be built (one for each hemisphere).

Space based instruments would provide ideal conditions, but a very high cost of operation. Some day there may be a Space Schmidt Telescope (or Moon Schmidt Telescope ...? ), but how soon and how large mainly depends on the astronomical community's will to support such a project.

\section{Bibllography:}

Angel 1983: ' Implementation and use of wide fields in very large telescopes ' Astronomy with Schmidt type telescopes - IAU Colloquium \# 78 - Cappacioli ed. 1983. p.549

Quebatte et al. 1986: ' Grid processing of large photographic plates' - The Messenger Issue \# 46, p.7 
Discussion:

RISTEMAKER noise in the plates? MAURY photographic plate is a function of gamma (the local contrast) divided by the RMS granularity. One has to limit the contrast of the emulsion if a decent dynamic range is needed and granularity is a function of the crystal size. But speed is also a function of crystal size so every emulsion is a compromise between maximum allowable exposure time and "noise" in the emulsion. 